\title{
Global Stability for a Predator-Prey Model with Dispersal among Patches
}

\author{
Yang Gao ${ }^{1,2}$ and Shengqiang Liu ${ }^{1}$ \\ ${ }^{1}$ Academy of Fundamental and Interdisciplinary Science, Harbin Institute of Technology, Harbin 150080, China \\ ${ }^{2}$ Department of Mathematics, Daqing Normal University, Daqing, Heilongjiang 163712, China
}

Correspondence should be addressed to Shengqiang Liu; sqliu@hit.edu.cn

Received 19 January 2014; Accepted 6 February 2014; Published 12 March 2014

Academic Editor: Weiming Wang

Copyright (c) 2014 Y. Gao and S. Liu. This is an open access article distributed under the Creative Commons Attribution License, which permits unrestricted use, distribution, and reproduction in any medium, provided the original work is properly cited.

\begin{abstract}
We investigate a predator-prey model with dispersal for both predator and prey among $n$ patches; our main purpose is to extend the global stability criteria by Li and Shuai (2010) on a predator-prey model with dispersal for prey among $n$ patches. By using the method of constructing Lyapunov functions based on graph-theoretical approach for coupled systems, we derive sufficient conditions under which the positive coexistence equilibrium of this model is unique and globally asymptotically stable if it exists.
\end{abstract}

\section{Introduction}

In the literature of predator-prey population systems, both continuous reaction-diffusion systems and discrete patchy models are used to study the spatial heterogeneity $[1,2]$; patchy models are often used to describe directed movement of population among niches or migration among habitats. It is naturally interesting problem to consider how the dispersal or migration of predator and prey influences the global dynamics of the interacting ecological system; thus patchy predator-prey model received lots of attentions [1,3-6].

Since the discrete patchy models usually involve highdimensional system, it is rather mathematically challenging to study the uniqueness and stability of the positive equilibrium of the predator-prey patchy models, and the available global dynamics criteria in the literatures mainly focus on the special case of two-patch [3] or on the permanence and existence of periodic solutions [4-6].

Recently, $\mathrm{Li}$ and Shuai [7] considered the following predator-prey model with dispersal for prey among $n$-patch:

$$
\begin{gathered}
\dot{x}_{i}=x_{i}\left(r_{i}-b_{i} x_{i}-e_{i} y_{i}\right)+\sum_{j=1}^{n} d_{i j}^{x}\left(x_{j}-\alpha_{i j}^{x} x_{i}\right), \\
\dot{y}_{i}=y_{i}\left(-\gamma_{i}-\delta_{i} y_{i}+\varepsilon_{i} x_{i}\right), \quad i=1, \ldots, n .
\end{gathered}
$$

Here, $x_{i}, y_{i}$ denote the densities of prey and predators on the patch $i$, respectively. The parameters $r_{i}, b_{i}$ and $\gamma_{i}, \delta_{i}$ in the model are nonnegative constants. What is more, the parameters $e_{i}$ and $\varepsilon_{i}$ in the model are positive constants. Constant $d_{i j}^{x}$ is the dispersal rate of the prey from patch $j$ to patch $i$ and constants $\alpha_{i j}^{x}$ can be selected to represent different boundary conditions in the continuous diffusion case.

In [7], the authors studied the global stability of the coexistence equilibrium of system (1), by considering (1) as a coupled $n$ predator-prey submodels on networks. Using results from graph theory and a developed systematic approach that allows one to construct global Lyapunov functions for largescale coupled systems from building blocks of individual vertex systems, Li and Shuai [7] obtain the following sharp results for (1).

Proposition 1 (see [7, Theorem 6.1]). Assume that $\left(d_{i j}^{x}\right)_{n \times n}$ is irreducible. If there exists $k$ such that $b_{k}>0$ or $\delta_{k}>0$, then, whenever a positive equilibrium $E_{*}$ exists in (1), it is unique and globally asymptotically stable in the positive cone $R_{2 n}^{+}$.

Although well-improved results have been seen in the above work on dispersal predator-prey model, such models are not well studied yet in the sense that model (1) assumes no dispersal for predator, which is not realistic in many cases $[1,3]$. Thus it is interesting for us to consider the global 
stability of the positive equilibrium for predator-prey model with dispersal for both predator and prey.

Motivated by the above work in [7], in this paper we generalize model (1) into the following predator-prey model with dispersal for both predator and prey:

$$
\begin{array}{r}
\dot{x}_{i}=x_{i}\left(r_{i}-b_{i} x_{i}-e_{i} y_{i}\right)+\sum_{j=1}^{n} d_{i j}^{x}\left(x_{j}-\alpha_{i j}^{x} x_{i}\right) \\
\dot{y}_{i}=y_{i}\left(-\gamma_{i}-\delta_{i} y_{i}+\varepsilon_{i} x_{i}\right)+\sum_{j=1}^{n} d_{i j}^{y}\left(y_{j}-\alpha_{i j}^{y} y_{i}\right), \\
i=1, \ldots, n .
\end{array}
$$

Here, the parameters $r_{i}, b_{i}, e_{i}, \gamma_{i}, \delta_{i}$, and $\varepsilon_{i}$ are defined the same as those in (1). The nonnegative constants $d_{i j}^{y}, \alpha_{i j}^{y}$, and $d_{i j}^{y}$ are the dispersal rate of the predators from patch $j$ to patch $i$, and $\alpha_{i j}^{y}$ represents the different boundary conditions in the continuous diffusion case. Clearly, when $d_{i j}^{y}=0$ for all $i, j=1, \ldots, n$, model (2) directly reduces to (1); thus our model (2) directly extends model (1) in [7].

The main purpose of this paper is to obtain the global stability for the coexistence equilibrium of (2). We will engage the techniques of constructing Lyapunov function based on graph-theory which were well developed by Li et al. in [7-9]; we refer to [10-12] for recent applications. Our study seems to be the first attempt in applying the network method for coupled network systems of differential equations to address the predator-prey system with dispersal for both predator and prey among patches. Networked method has been extensively investigated in the several fields. For example, multiagent systems can be seen as complicated network systems. A lot of researchers take their interest in flocking and consensus of the multiagent systems [13-17]. What is more, neural network systems can be seen as complicated network systems. Over the past few decades, various neural network models have been extensively investigated [18-20].

A mathematical description of a network is a directed graph consisting of vertices and directed arcs connecting them. At each vertex, the local dynamics are given by a system of differential equations called the vertex system. The directed arcs indicate interconnections and interactions among vertex systems.

A digraph $G$ with $n$ vertices for the system (2) can be constructed as follows. Each vertex represents a patch and $(j, i) \in E(G)$ if and only if $d_{i j}^{x}, d_{i j}^{y}>0$. At each vertex of $G$, the vertex dynamics is described by a predator-prey system. The coupling among these predator-prey systems is provided by dispersal of predator and prey among patches.

This paper is organized as follows. In the next section, we introduce preliminaries results on graph-theory based on coupled network models. In Section 3, we obtain the main result of system (2). This is followed by a brief conclusion section.

\section{Preliminaries}

In this section, we will list some definitions and Theorems that we will use in the later sections.

A directed graph or digraph $G=(V, E)$ contains a set $V=$ $\{1,2, \ldots, n\}$ of vertices and a set $E$ of $\operatorname{arcs}(i, j)$ leading from initial vertex $i$ to terminal vertex $j$. A subgraph $H$ of $G$ is said to be spanning if $H$ and $G$ have the same vertex set. A digraph $G$ is weighted if each $\operatorname{arc}(j, i)$ is assigned a positive weight. $a_{i j}>0$ if and only if there exists an arc from vertex $j$ to vertex $i$ in $G$.

The weight $w(H)$ of a subgraph $H$ is the product of the weights on all its arcs. A directed path $P$ in $G$ is a subgraph with distinct vertices $i_{1}, i_{2}, \ldots, i_{m}$ such that its set of arcs is $\left\{\left(i_{k}, i_{k+1}\right): k=1,2, \ldots, m\right\}$. If $i_{m}=i_{1}$, we call $P$ a directed cycle.

A connected subgraph $T$ is a tree if it contains no cycles, directed or undirected.

A tree $T$ is rooted at vertex $i$, called the root, if $i$ is not a terminal vertex of any arcs, and each of the remaining vertices is a terminal vertex of exactly one arc. A subgraph $Q$ is unicyclic if it is a disjoint union of rooted trees whose roots form a directed cycle.

Given a weighted digraph $G$ with $n$ vertices, define the weight matrix $A=\left(a_{i j}\right)_{n \times n}$ whose entry $a_{i j}$ equals the weight of $\operatorname{arc}(j, i)$ if it exists, and 0 otherwise. For our purpose, we denote a weighted digraph as $(G, A)$. A digraph $G$ is strongly connected if for any pair of distinct vertices, there exists a directed path from one to the other. A weighted digraph $(G, A)$ is strongly connected if and only if the weight matrix $A$ is irreducible.

The Laplacian matrix of $(G, A)$ is denoted by $L$. Let $c_{i}$ denote the cofactor of the $i$ th diagonal element of $L$. The following results are listed as follows from [7].

Proposition 2 (see [7]). Assume $n \geq 2$. Then

$$
c_{i}=\sum_{\mathbf{T} \in T_{i}} w(\mathbf{T}),
$$

where $T_{i}$ is the set of all spanning trees $\mathbf{T}$ of $(G, A)$ that are rooted at vertex $i$, and $w(T)$ is the weight of $T$. In particular, if $(G, A)$ is strongly connected, then $c_{i}>0$ for $1 \leq i \leq n$.

Theorem 3 (see [7]). Assume $n \geq 2$. Let $c_{i}$ be given in Proposition 2. Then the following identity holds:

$$
\sum_{i, j=1}^{n} c_{i} a_{i j} F_{i j}\left(x_{i}, x_{j}\right)=\sum_{\mathrm{Q} \in \mathbf{Q}} w(Q) \sum_{(s, r) \in E\left(C_{Q}\right)} F_{r s}\left(x_{r}, x_{s}\right),
$$

where $F_{i j}\left(x_{i}, x_{j}\right), 1 \leq i, j \leq n$, are arbitrary functions, $\mathbf{Q}$ is the set of all spanning unicyclic graphs of $(G, A), w(Q)$ is the weight of $Q$, and $C_{Q}$ denotes the directed cycle of $Q$.

Given a network represented by digraph $G$ with $n$ vertices, $n \geq 2$, a coupled system can be built on $G$ by assigning each vertex its own internal dynamics and then coupling these vertex dynamics based on directed arcs in $G$. Assume that 
each vertex dynamics is described by a system of differential equations

$$
u_{i}^{\prime}=f_{i}\left(t, u_{i}\right) \text {, }
$$

where $u_{i} \in \mathbf{R}^{\mathbf{m}_{\mathbf{i}}}$ and $f_{i}: \mathbf{R} \times \mathbf{R}^{\mathbf{m}_{\mathbf{i}}} \rightarrow \mathbf{R}^{\mathbf{m}_{\mathbf{i}}}$. Let $g_{i j}: \mathbf{R} \times \mathbf{R}^{\mathbf{m}_{\mathbf{i}}} \times$ $\mathbf{R}^{\mathbf{m}_{\mathbf{j}}} \rightarrow \mathbf{R}^{\mathbf{m}_{\mathbf{i}}}$ represent the influence of vertex $j$ on vertex $i$, and let $g_{i j} \equiv 0$ if there exists no arc from $j$ to $i$ in $G$. Then we obtain the following coupled system on graph $G$ :

$$
u_{i}^{\prime}=f_{i}\left(t, u_{i}\right)+\sum_{j=1}^{n} g_{i j}\left(t, u_{i}, u_{j}\right), \quad i=1,2, \ldots, n .
$$

Here functions $f_{i}, g_{i j}$ are such that initial-value problems have unique solutions.

We assume that each vertex system has a globally stable equilibrium and possesses a global Lyapunov function $V_{i}$.

Theorem 4 (see [7]). Assume that the following assumptions are satisfied.

(1) There exist functions $V_{i}\left(t, u_{i}\right), F_{i j}\left(t, u_{i}, u_{j}\right)$ and constants $a_{i j} \geq 0$ such that

$$
\dot{V}_{i}\left(t, u_{i}\right) \leq \sum_{i, j=1}^{n} a_{i j} F_{i j}\left(t, u_{i}, u_{j}\right), \quad t>0, u_{i} \in D_{i} .
$$

(2) Along each directed cycle $C$ of the weighted digraph $(G, A), A=\left(a_{i j}\right)$,

$$
\sum_{(s, r) \in E(C)} F_{r s}\left(t, u_{r}, u_{s}\right) \leq 0 .
$$

(3) Constants $c_{i}$ are given by the cofactor of the ith diagonal element of $L$.

Then the function $V(t, u)=\sum_{i=1}^{n} c_{i} V_{i}\left(t, u_{i}\right)$ satisfies $\dot{V}(t, u) \leq 0$ for $t>0, u \in D$; namely, $V$ is a Lyapunov function for the system (6).

\section{Main Results}

In this section, the stability for the positive equilibrium of the $n$-patch predator-prey model (2) is considered. We regard (2) as a coupled system on a network. Using a Lyapunov function for the $n$-patch predator-prey model with dispersal and Theorem 4 of Section 2, we will establish that a positive equilibrium of the $n$-patch predator-prey model (2) with dispersal is globally asymptotically stable in $\mathbf{R}_{+}^{2 n}$ as long as it exists.

First of all, we will give a lemma for the system (2).

Lemma 5. The set $\mathbf{R}_{+}^{2 n}$ is the positive invariant set for the system (2).

The next Theorem gives the globally asymptotically stable condition for the positive equilibrium of the system (2).

Theorem 6. Assume that a positive equilibrium $E^{*}=$ $\left(x_{1}^{*}, y_{1}^{*}, x_{2}^{*}, y_{2}^{*}, \ldots, x_{n}^{*}, y_{n}^{*}\right)$ exists for the system (2) and the following assumptions hold.
(1) Dispersal matrixes $\left(d_{i j}^{x}\right)_{n \times n},\left(d_{i j}^{y}\right)_{n \times n}$ are irreducible; moreover there exists $k$ such that $b_{k}>0$ or $\delta_{k}>0$.

(2) There exists nonnegative constant $\lambda$ such that $\lambda$. $d_{i j}^{x} \varepsilon_{i} x_{j}^{*}=d_{i j}^{y} e_{i} y_{j}^{*}$ for $1 \leq i, j \leq n$, or $d_{i j}^{x} \varepsilon_{i} x_{j}^{*}=$ $\lambda \cdot d_{i j}^{y} e_{i} y_{j}^{*}$ for $1 \leq i, j \leq n$.

Then, the positive equilibrium $E^{*}$ is unique and globally asymptotically stable in $R_{+}^{2 n}$.

Proof. Let

$$
\begin{gathered}
Z_{i}^{1}\left(x_{i}, y_{i}\right)=r_{i}-b_{i} x_{i}-e_{i} y_{i}, \\
Z_{i}^{2}\left(x_{i}, y_{i}\right)=-\gamma_{i}-\delta_{i} y_{i}+\varepsilon_{i} x_{i} .
\end{gathered}
$$

In the sequel, we have

$$
\begin{aligned}
& Z_{i}^{1}\left(x_{i}^{*}, y_{i}^{*}\right)=-\frac{1}{x_{i}^{*}} \sum_{j=1}^{n} d_{i j}^{x}\left(x_{j}^{*}-\alpha_{i j}^{x} x_{i}^{*}\right), \\
& Z_{i}^{2}\left(x_{i}^{*}, y_{i}^{*}\right)=-\frac{1}{y_{i}^{*}} \sum_{j=1}^{n} d_{i j}^{y}\left(y_{j}^{*}-\alpha_{i j}^{y} y_{i}^{*}\right) .
\end{aligned}
$$

Set Lyapunov functions as

$$
\begin{aligned}
V_{i}\left(x_{i}, y_{i}\right)= & \varepsilon_{i}\left(x_{i}-x_{i}^{*}-x_{i}^{*} \ln \frac{x_{i}}{x_{i}^{*}}\right) \\
& +e_{i}\left(y_{i}-y_{i}-y_{i}^{*} \ln \frac{y_{i}}{y_{i}^{*}}\right) .
\end{aligned}
$$

Direct differentiating $V_{i}$ along the system (2), we have

$$
\begin{aligned}
\dot{V}_{i}\left(x_{i}, y_{i}\right)= & \varepsilon_{i}\left(x_{i}-x_{i}^{*}\right)\left[Z_{i}^{1}\left(x_{i}, y_{i}\right)-Z_{i}^{1}\left(x_{i}^{*}, y_{i}^{*}\right)\right] \\
& +e_{i}\left(y_{i}-y_{i}^{*}\right)\left[Z_{i}^{2}\left(x_{i}, y_{i}\right)-Z_{i}^{2}\left(x_{i}^{*}, y_{i}^{*}\right)\right] \\
& +\varepsilon_{i}\left(x_{i}-x_{i}^{*}\right) Z_{i}^{1}\left(x_{i}^{*}, y_{i}^{*}\right) \\
& +\frac{\varepsilon_{i}\left(x_{i}-x_{i}^{*}\right)}{x_{i}^{*}} \sum_{j=1}^{n} d_{i j}^{x}\left(x_{j}-\alpha_{i j}^{x} x_{i}\right) \\
& +e_{i}\left(y_{i}-y_{i}^{*}\right) Z_{i}^{2}\left(x_{i}^{*}, y_{i}^{*}\right) \\
& +\frac{e_{i}\left(y_{i}-y_{i}^{*}\right)}{y_{i}^{*}} \sum_{j=1}^{n} d_{i j}^{y}\left(y_{j}-\alpha_{i j}^{y} y_{i}\right) \\
= & \varepsilon_{i}\left(x_{i}-x_{i}^{*}\right)\left[Z_{i}^{1}\left(x_{i}, y_{i}\right)-Z_{i}^{1}\left(x_{i}^{*}, y_{i}^{*}\right)\right] \\
& +e_{i}\left(y_{i}-y_{i}^{*}\right)\left[Z_{i}^{2}\left(x_{i}, y_{i}\right)-Z_{i}^{2}\left(x_{i}^{*}, y_{i}^{*}\right)\right] \\
& +\sum_{j=1}^{n} d_{i j}^{x} \varepsilon_{i} x_{j}^{*} F_{i j}^{x}\left(x_{i}, x_{j}\right)+\sum_{j=1}^{n} d_{i j}^{y} e_{i} y_{j}^{*} F_{i j}^{y}\left(y_{i}, y_{j}\right)
\end{aligned}
$$




$$
\begin{aligned}
= & -\varepsilon_{i} b_{i}\left(x_{i}-x_{i}^{*}\right)^{2}-\varepsilon_{i}\left(x_{i}-x_{i}^{*}\right) e_{i}\left(y_{i}-y_{i}^{*}\right) \\
& -e_{i} \delta_{i}\left(y_{i}-y_{i}^{*}\right)^{2}+e_{i}\left(y_{i}-y_{i}^{*}\right) \varepsilon_{i}\left(x_{i}-x_{i}^{*}\right) \\
& +\sum_{j=1}^{n} d_{i j}^{x} \varepsilon_{i} x_{j}^{*} F_{i j}^{x}\left(x_{i}, x_{j}\right)+\sum_{j=1}^{n} d_{i j}^{y} e_{i} y_{j}^{*} F_{i j}^{y}\left(y_{i}, y_{j}\right) \\
= & -\varepsilon_{i} b_{i}\left(x_{i}-x_{i}^{*}\right)^{2}-e_{i} \delta_{i}\left(y_{i}-y_{i}^{*}\right)^{2} \\
& +\sum_{j=1}^{n} d_{i j}^{x} \varepsilon_{i} x_{j}^{*} F_{i j}^{x}\left(x_{i}, x_{j}\right)+\sum_{j=1}^{n} d_{i j}^{y} e_{i} y_{j}^{*} F_{i j}^{y}\left(y_{i}, y_{j}\right),
\end{aligned}
$$

where

$$
\begin{aligned}
& F_{i j}^{x}\left(x_{i}, x_{j}\right)=\frac{x_{j}}{x_{j}^{*}}-\frac{x_{i}}{x_{i}^{*}}+1-\frac{x_{i}^{*} x_{j}}{x_{i} x_{j}^{*}}, \\
& F_{i j}^{y}\left(y_{i}, y_{j}\right)=\frac{y_{j}}{y_{j}^{*}}-\frac{y_{i}}{y_{i}^{*}}+1-\frac{y_{i}^{*} y_{j}}{y_{i} y_{j}^{*}} .
\end{aligned}
$$

Set $a_{i j}^{x}=d_{i j}^{x} \varepsilon_{i} x_{j}^{*}, b_{i j}^{y}=d_{i j}^{y} e_{i} y_{j}^{*}, A=\left(a_{i j}^{x}\right)_{n \times n}$, and $B=\left(b_{i j}^{y}\right)_{n \times n}$. One has

$$
G_{i}^{x}\left(x_{i}\right)=-\frac{x_{i}}{x_{i}^{*}}+\ln \frac{x_{i}}{x_{i}^{*}}, \quad G_{i}^{y}\left(y_{i}\right)=-\frac{y_{i}}{y_{i}^{*}}+\ln \frac{y_{i}}{y_{i}^{*}} .
$$

Next, we have two cases to consider.

Case I. $d_{i j}^{x} \varepsilon_{i} x_{j}^{*}=\lambda \cdot d_{i j}^{y} e_{i} y_{j}^{*}$ for $1 \leq i, j \leq n$.

Case II. $\lambda \cdot d_{i j}^{x} \varepsilon_{i} x_{j}^{*}=d_{i j}^{y} e_{i} y_{j}^{*}$ for $1 \leq i, j \leq n$.

For Case I, from the fact that $a_{i j}^{x}=d_{i j}^{x} \varepsilon_{i} x_{j}^{*}$ and $b_{i j}^{y}=$ $d_{i j}^{y} e_{i} y_{j}^{*}$, we obtain that $a_{i j}^{x}=\lambda b_{i j}^{y}$; thus $A=\lambda \cdot B$. Then we obtain that

$$
\begin{aligned}
\dot{V}_{i}\left(x_{i}, y_{i}\right) \leq & -\varepsilon_{i} b_{i}\left(x_{i}-x_{i}^{*}\right)^{2}-e_{i} \delta_{i}\left(y_{i}-y_{i}^{*}\right)^{2} \\
& +\sum_{j=1}^{n} a_{i j}^{x}\left(G_{i}^{x}\left(x_{i}\right)-G_{j}^{x}\left(x_{j}\right)\right) \\
& +\sum_{j=1}^{n} a_{i j}^{x}\left(1-\frac{x_{i}^{*} x_{j}}{x_{i} x_{j}^{*}}+\ln \frac{x_{i}^{*} x_{j}}{x_{i} x_{j}^{*}}\right) \\
& +\sum_{j=1}^{n} b_{i j}^{y}\left(G_{i}^{y}\left(y_{i}\right)-G_{j}^{y}\left(y_{j}\right)\right) \\
& +\sum_{j=1}^{n} b_{i j}^{y}\left(1-\frac{y_{i}^{*} y_{j}}{y_{i} y_{j}^{*}}+\ln \frac{y_{i}^{*} y_{j}}{y_{i} y_{j}^{*}}\right)
\end{aligned}
$$

$$
\begin{aligned}
\leq & -\varepsilon_{i} b_{i}\left(x_{i}-x_{i}^{*}\right)^{2}-e_{i} \delta_{i}\left(y_{i}-y_{i}^{*}\right)^{2} \\
& +\lambda \sum_{j=1}^{n} b_{i j}^{y}\left(G_{i}^{x}\left(x_{i}\right)-G_{j}^{x}\left(x_{j}\right)\right) \\
& +\lambda \sum_{j=1}^{n} b_{i j}^{y}\left(1-\frac{x_{i}^{*} x_{j}}{x_{i} x_{j}^{*}}+\ln \frac{x_{i}^{*} x_{j}}{x_{i} x_{j}^{*}}\right) \\
& +\sum_{j=1}^{n} b_{i j}^{y}\left(G_{i}^{y}\left(y_{i}\right)-G_{j}^{y}\left(y_{j}\right)\right) \\
& +\sum_{j=1}^{n} b_{i j}^{y}\left(1-\frac{y_{i}^{*} y_{j}}{y_{i} y_{j}^{*}}+\ln \frac{y_{i}^{*} y_{j}}{y_{i} y_{j}^{*}}\right) .
\end{aligned}
$$

Let $c_{i}^{y}$ denote the cofactor of the $i$ th diagonal element of the matrix $B$. From the irreducible character of matrix $B$, we have $c_{i}^{y}>0$.

Furthermore, set Lyapunov functions as

$$
\begin{aligned}
V(x, y) & =V\left(x_{1}, y_{1}, \ldots, x_{n}, y_{n}\right) \\
& =\sum_{i=1}^{n} c_{i}^{y} V_{i}^{x}\left(x_{i}\right)+\sum_{i=1}^{n} c_{i}^{y} V_{i}^{y}\left(y_{i}\right) .
\end{aligned}
$$

Then differentiating $V$ along the solution of the system (2), we obtain that

$$
\begin{aligned}
\dot{V}(x, y) \leq & -\sum_{i=1}^{n} c_{i}^{y} \varepsilon_{i} b_{i}\left(x_{i}-x_{i}^{*}\right)^{2}-\sum_{i=1}^{n} c_{i}^{y} e_{i} \delta_{i}\left(y_{i}-y_{i}^{*}\right)^{2} \\
& +\sum_{i, j=1}^{n} \lambda b_{i j}^{y} c_{i}^{y}\left(G_{i}^{x}\left(x_{i}\right)-G_{j}^{x}\left(x_{j}\right)\right) \\
& +\sum_{i, j=1}^{n} b_{i j}^{y} c_{i}^{y}\left(G_{i}^{y}\left(y_{i}\right)-G_{j}^{y}\left(y_{j}\right)\right) .
\end{aligned}
$$

Let $G$ represent the directed graph associated with matrix $B$. Then $G$ has vertices $1,2, \ldots, n$ with a directed $\operatorname{arc}(k, j)$ from $k$ to $j$ if and only if $b_{k j}^{y} \neq 0$. Then $E(G)$ is the set of all directed arcs of $G$. By Kirchhoff's Matrix-Tree Theorem (see Proposition 2) we know that $v_{k}=C_{k k}$ can be expressed as a sum of weights of all directed spanning subtrees $T$ of $G$ that are rooted at vertex $k$. Thus, each term in $v_{k} a_{k j}$ is the weight $\omega(Q)$ of a unicyclic subgraph $Q$ of $G$ obtained from such a tree $T$ by adding a directed $\operatorname{arc}(k, j)$ from the root $k$ to vertex $j$. Because the arc $(k, j)$ is a part of the unique cycle $C Q$ of $Q$ and that the same unicyclic graph $Q$ can be formed when each arc of $C Q$ is added to a corresponding rooted tree $T$, then the double sum can be expressed as a sum over all unicyclic subgraphs $Q$ containing vertices $1,2, \ldots, n$. 
Therefore, following from the irreducible character of matrix $B$ and Theorem 2.3 in [7], we obtain

$$
\begin{aligned}
& \sum_{i, j=1}^{n} \lambda b_{i j}^{y} c_{i}^{y}\left(G_{i}^{x}\left(x_{i}\right)-G_{j}^{x}\left(x_{j}\right)\right)=0 \\
& \sum_{i, j=1}^{n} b_{i j}^{y} c_{i}^{y}\left(G_{i}^{y}\left(y_{i}\right)-G_{j}^{y}\left(y_{j}\right)\right)=0 .
\end{aligned}
$$

Combining with the fact that $1-a+\ln a \leq 0$, therefore we have

$$
\dot{V}(x, y) \leq 0 .
$$

When we consider $\dot{V}(x, y)=0$, by condition 1 , there exists $k \in N_{+}$such that

$$
\left(x_{k}-x_{k}^{*}\right)^{2}=0 \quad \text { or } \quad\left(y_{k}-y_{k}^{*}\right)^{2}=0 .
$$

It means that $x_{k}=x_{k}^{*}$ or $y_{k}=y_{k}^{*}$.

If $i$ and $k$ can be connected with an arc from $k$ to $i$ in $G$, then we have $a_{i k}^{y}>0$ and $b_{i k}^{y}>0$. Furthermore,

$$
\begin{aligned}
& 1-\frac{x_{i}^{*} x_{k}}{x_{i} x_{k}^{*}}+\ln \frac{x_{i}^{*} x_{k}}{x_{i} x_{k}^{*}}=0, \\
& 1-\frac{y_{i}^{*} y_{k}}{y_{i} y_{k}^{*}}+\ln \frac{y_{i}^{*} y_{k}}{y_{i} y_{k}^{*}}=0 .
\end{aligned}
$$

Because of $1-a+\ln a \leq 0$ and $1-a+\ln a=0$, $\Leftrightarrow a=0$. we deduce that

$$
\frac{x_{i}}{x_{i}^{*}}=\frac{x_{k}}{x_{k}^{*}}, \quad \frac{y_{i}}{y_{i}^{*}}=\frac{y_{k}}{y_{k}^{*}} .
$$

From $x_{k}=x_{k}^{*}$, or $y_{k}=y_{k}^{*}$, we obtain that $x_{i}=x_{i}^{*}$ and $y_{i} / y_{i}^{*}=$ $y_{k} / y_{k}^{*}$ or $y_{i}=y_{i}^{*}$ and $x_{i} / x_{i}^{*}=x_{k} / x_{k}^{*}$.

By condition 1 and the definition of matrixes $A, B$, we get that $B$ are irreducible. By strong connectivity of $G$, there exists a directed path $P$ from any $i$ to $k$. Then we have that, for any $i=1,2, \ldots, n$, there must be

$$
x_{i}=x_{i}^{*}, \quad \frac{y_{i}}{y_{i}^{*}}=\mu, \quad \mu \geq 0,
$$

or for any $i=1,2, \ldots, n$, there must be

$$
y_{i}=y_{i}^{*}, \quad \frac{x_{i}}{x_{i}^{*}}=\mu, \quad \mu \geq 0 .
$$

Next, we will prove that the largest compact invariant subset of $\{(x, y) \mid \dot{V}(x, y)=0\}$ is the singleton $\left\{E^{*}\right\}$.

We only consider the case that

$$
x_{i}=x_{i}^{*}, \quad \frac{y_{i}}{y_{i}^{*}}=\mu, \quad i=1,2, \ldots, n, \mu \geq 0 .
$$

The case that

$$
y_{i}=y_{i}^{*}, \quad \frac{x_{i}}{x_{i}^{*}}=\mu, \quad i=1,2, \ldots, n, \mu \geq 0
$$

is similar to this case. So we omit it.
If $\mu=0$, we have $y_{i}=0$ for any $i=1,2, \ldots, n$, and then we have

$$
x_{i}^{*}\left(r_{i}-b_{i} x_{i}^{*}\right)+\sum_{j=1}^{n} d_{i j}^{x}\left(x_{j}^{*}-\alpha_{i j}^{x} x_{i}^{*}\right)=0,
$$

which contradicts to the fact that

$$
x_{i}^{*}\left(r_{i}-b_{i} x_{i}^{*}-e_{i} y_{i}^{*}\right)+\sum_{j=1}^{n} d_{i j}^{x}\left(x_{j}^{*}-\alpha_{i j}^{x} x_{i}^{*}\right)=0 .
$$

If $\mu>0$ and $\mu \neq 1$, we have $y_{i}=\mu y_{i}^{*}$ for any $i=1,2, \ldots, n$, and then we have

$$
x_{i}^{*}\left(r_{i}-b_{i} x_{i}^{*}-e_{i} \mu y_{i}^{*}\right)+\sum_{j=1}^{n} d_{i j}^{x}\left(x_{j}^{*}-\alpha_{i j}^{x} x_{i}^{*}\right)=0,
$$

which also contradicts to the fact that

$$
x_{i}^{*}\left(r_{i}-b_{i} x_{i}^{*}-e_{i} y_{i}^{*}\right)+\sum_{j=1}^{n} d_{i j}^{x}\left(x_{j}^{*}-\alpha_{i j}^{x} x_{i}^{*}\right)=0 .
$$

Therefore, we obtain that $\mu=1$, which means

$$
x_{i}=x_{i}^{*}, \quad y_{i}=y_{i}^{*}, \quad i=1,2, \ldots, n .
$$

Namely, we get that the largest compact invariant subset of $\{(x, y) \mid \dot{V}(x, y)=0\}$ is the singleton $\left\{E^{*}\right\}$. Therefore, by the LaSalle Invariance Principle ([21]), $E^{*}$ is globally asymptotically stable in $\mathbf{R}_{+}^{2 n}$.

With the similar arguments to the Case I, we can prove that $E^{*}$ is globally asymptotically stable in $\mathbf{R}_{+}^{2 n}$ for Case II. This completes the proof.

Remark 7. Theorem 6 is applicable to model (1): consider model (2) with $d_{i j}^{y}=0, i, j=1, \ldots, n$, and let $\lambda=0$; thus Theorem 6 directly reduces to Proposition 1 by Li and Shuai [7] for (1).

By Theorem 6 and similar arguments to Remark 7, we directly have the following global stability theorem for the predator-prey model with discrete dispersal of predator among patches.

Corollary 8. Consider the model

$$
\begin{gathered}
\dot{x}_{i}=x_{i}\left(r_{i}-b_{i} x_{i}-e_{i} y_{i}\right), \\
\dot{y}_{i}=y_{i}\left(-\gamma_{i}-\delta_{i} y_{i}+\varepsilon_{i} x_{i}\right)+\sum_{j=1}^{n} d_{i j}^{y}\left(y_{j}-\alpha_{i j}^{y} y_{i}\right), \\
i=1, \ldots, n .
\end{gathered}
$$

Assume that the matrix $\left(d_{i j}^{y}\right)_{n \times n}$ is irreducible. If there exists $k$ such that $b_{k}>0$ or $\delta_{k}>0$; then, whenever a positive equilibrium $E_{*}$ exists in (32), it is unique and globally asymptotically stable in the positive cone $R_{+}^{2 n}$. 


\section{Discussion}

In this paper, we generalize the model of the $n$-patch predator-prey model of [7] to the general model (2) that both the prey and the predator have dispersal among $n$ patches. Based on the network method for coupled systems of differential equations developed in [7-9], we prove that the positive equilibrium of (2) is globally asymptotically stable given some conditions on the coupling (see Theorem 6). Our main theorem generalizes Theorem 6.1 in [7] and our results also cover the other case of (2) in that only the predators disperse among patches.

Biologically, our result of Theorem 6 implies that if predator-prey system is dispersing among strongly connected patches (which is equivalent to the irreducibility of the dispersal matrixes of predator and prey) and if the system is permanent (which guarantees the existence of positive equilibrium), then the numbers of both predators and prey in each patches will eventually be stable at some corresponding positive values given the well-coupled dispersal (condition 2 of Theorem 6).

We remark that our Theorem 6 requires the extra condition 2 for the coupling dispersal coefficients and that the global dynamics for the coexistence equilibrium of (2) without condition 2 of Theorem 6 are still unclear. It remains an interesting future problem for the patchy dispersal predatorprey model.

\section{Conflict of Interests}

The authors declare that there is no conflict of interests regarding the publication of this paper.

\section{Acknowledgments}

The first author is supported by the Natural Science Foundation for Doctor of Daqing Normal University (no. 12ZR09). Shengqiang Liu is supported by the NNSF of China (no. 10601042), the Fundamental Research Funds for the Central Universities (no. HIT.NSRIF.2010052), and Program of Excellent Team in Harbin Institute of Technology.

\section{References}

[1] H. I. Freedman and Y. Takeuchi, "Global stability and predator dynamics in a model of prey dispersal in a patchy environment," Nonlinear Analysis: Theory, Methods \& Applications, vol. 13, no. 8, pp. 993-1002, 1989.

[2] J. D. Murry, Mathematical Biology, vol. 1-2, Springer, New York, NY, USA, 2002.

[3] Y. Kuang and Y. Takeuchi, "Predator-prey dynamics in models of prey dispersal in two-patch environments," Mathematical Biosciences, vol. 120, no. 1, pp. 77-98, 1994.

[4] J. Cui, "The effect of dispersal on permanence in a predatorprey population growth model," Computers \& Mathematics with Applications, vol. 44, no. 8-9, pp. 1085-1097, 2002.

[5] R. Xu, M. A. J. Chaplain, and F. A. Davidson, "Periodic solutions for a delayed predator-prey model of prey dispersal in two-patch environments," Nonlinear Analysis: Real World Applications, vol. 5, no. 1, pp. 183-206, 2004.

[6] L. Zhang and Z. Teng, "Permanence for a delayed periodic predator-prey model with prey dispersal in multi-patches and predator density-independent," Journal of Mathematical Analysis and Applications, vol. 338, no. 1, pp. 175-193, 2008.

[7] M. Y. Li and Z. Shuai, "Global-stability problem for coupled systems of differential equations on networks," Journal of Differential Equations, vol. 248, no. 1, pp. 1-20, 2010.

[8] H. Guo, M. Y. Li, and Z. Shuai, "A graph-theoretic approach to the method of global Lyapunov functions," Proceedings of the American Mathematical Society, vol. 136, no. 8, pp. 2793-2802, 2008.

[9] M. Y. Li and Z. Shuai, "Global stability of an epidemic model in a patchy environment," Canadian Applied Mathematics Quarterly, vol. 17, no. 1, pp. 175-187, 2009.

[10] H. Shu, D. Fan, and J. Wei, "Global stability of multi-group SEIR epidemic models with distributed delays and nonlinear transmission," Nonlinear Analysis: Real World Applications, vol. 13, no. 4, pp. 1581-1592, 2012.

[11] R. Sun and J. Shi, "Global stability of multigroup epidemic model with group mixing and nonlinear incidence rates," Applied Mathematics and Computation, vol. 218, no. 2, pp. 280286, 2011.

[12] J. Wang, J. Zu, X. Liu, G. Huang, and J. Zhang, "Global dynamics of a multi-group epidemic model with general relapse distribution and nonlinear incidence rate," The Journal of Biological Systems, vol. 20, no. 3, pp. 235-258, 2012.

[13] R. Olfati-Saber, "Flocking for multi-agent dynamic systems: algorithms and theory," IEEE Transactions on Automatic Control, vol. 51, no. 3, pp. 401-420, 2006.

[14] N. Moshtagh, A. Jadbabaie, and K. Daniilidis, "Distributed geodesic control laws for flocking of nonholonomic agents," in Proceedings of the 44th IEEE Conference on Decision and Control, and the European Control Conference (CDC-ECC '05), pp. 2835-2840, December 2005.

[15] R. A. Freeman, Y. Peng, and K. M. Lynch, "Distributed estimation and control of swarm formation statistics," in Proceedings of the American Control Conference, pp. 749-755, June 2006.

[16] Y. Hong, L. Gao, D. Cheng, and J. Hu, "Lyapunov-based approach to multiagent systems with switching jointly connected interconnection," IEEE Transactions on Automatic Control, vol. 52, no. 5, pp. 943-948, 2007.

[17] R. Olfati-Saber and J. S. Shamma, "Consensus filters for sensor networks and distributed sensor fusion," in Proceedings of the 44th IEEE Conference on Decision and Control, and the European Control Conference (CDC-ECC '05), pp. 6698-6703, December 2005.

[18] M. T. Hagan, H. B. Demuth, and M. H. Beale, Neural Network Design, China Machine, Beijing, China, 2002.

[19] Z. H. Zhou and C. G. Cao, Neural Network with Applications, Tsinghua University Press, Beijing, China, 2004.

[20] C. Hu, J. Yu, H. Jiang, and Z. Teng, "Exponential stabilization and synchronization of neural networks with time-varying delays via periodically intermittent control," Nonlinearity, vol. 23, no. 10, pp. 2369-2391, 2010.

[21] H. K. Khalil, Nonlinear Systems, Prentice Hall, 3rd edition, 2002. 


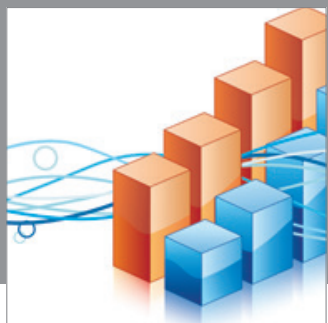

Advances in

Operations Research

mansans

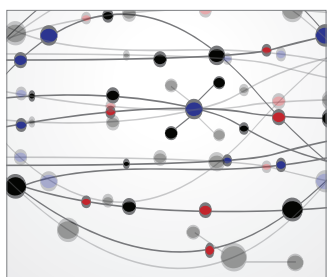

The Scientific World Journal
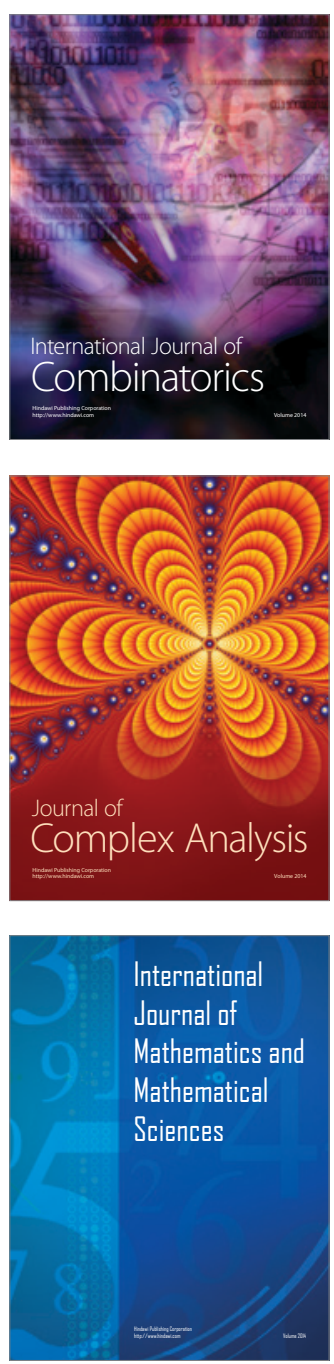
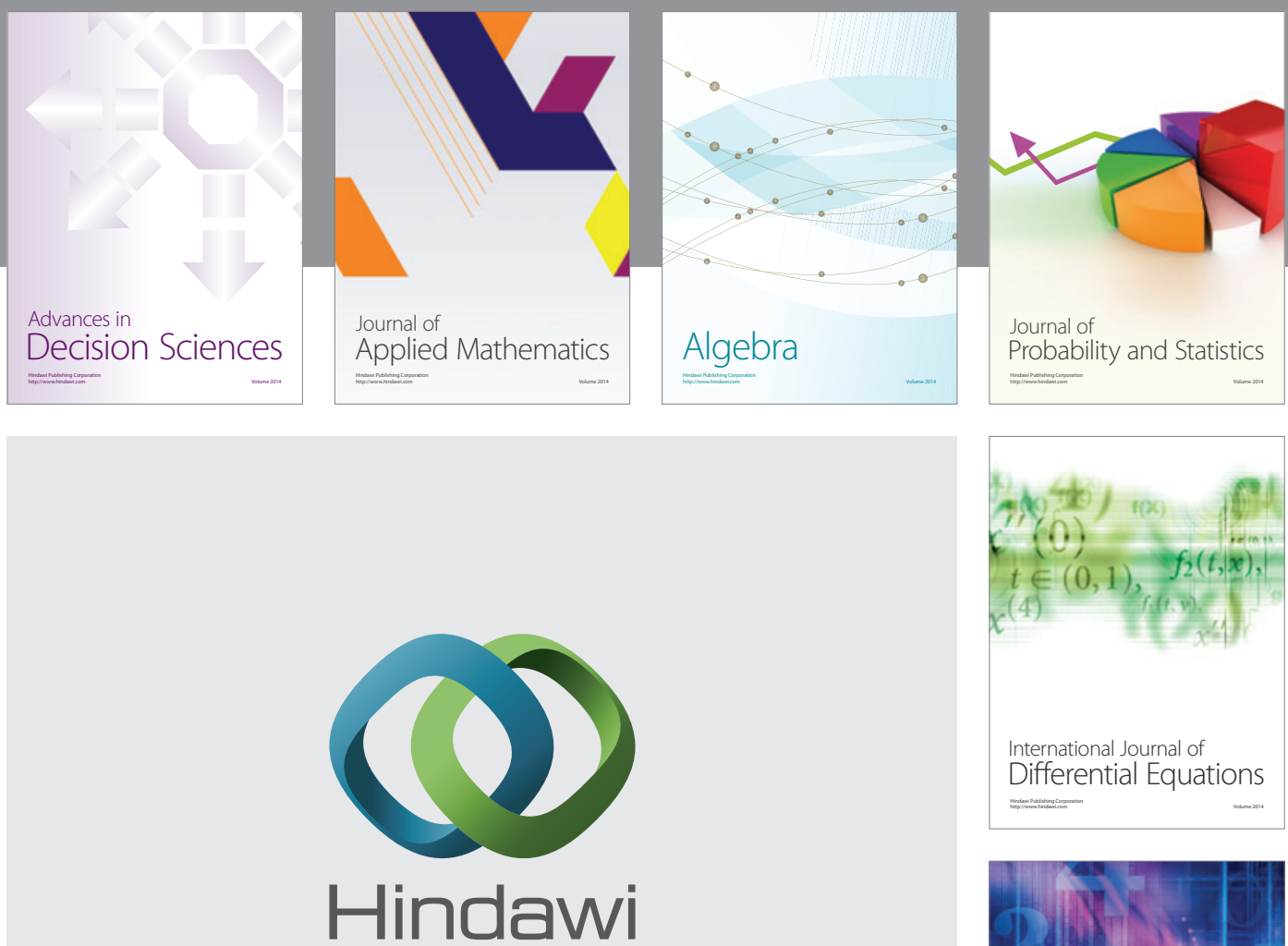

Submit your manuscripts at http://www.hindawi.com
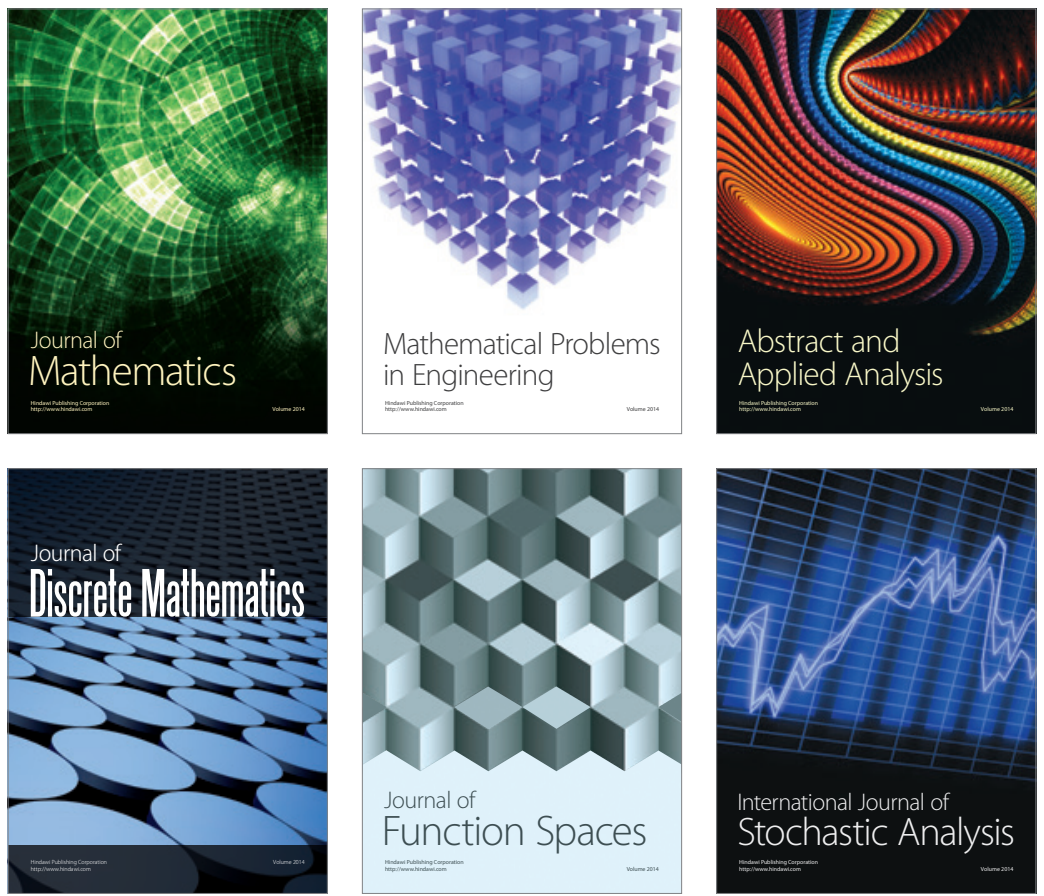

Journal of

Function Spaces

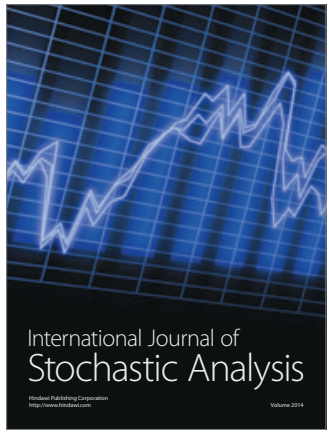

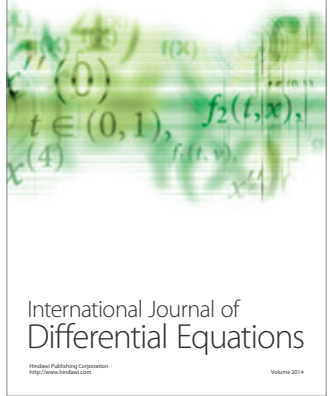
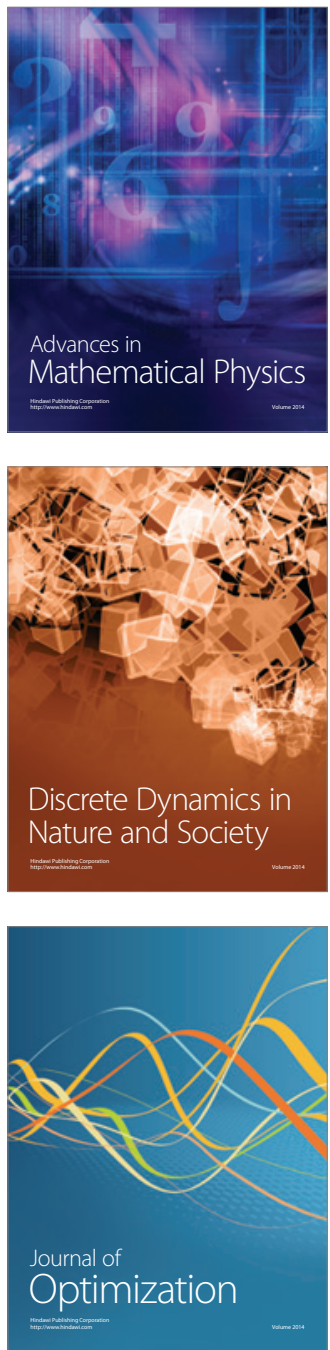